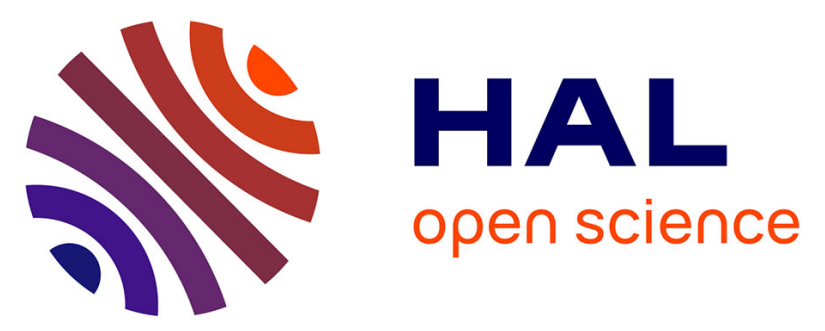

\title{
ON THE STATISTICAL PROPERTIES OF THE ELECTRONIC LEVELS OF SMALL METALLIC PARTICLES
}

J. Barojas, E. Cota, E. Blaisten-Barojas, J. Flores, P. Mello

\section{- To cite this version:}

J. Barojas, E. Cota, E. Blaisten-Barojas, J. Flores, P. Mello. ON THE STATISTICAL PROPERTIES OF THE ELECTRONIC LEVELS OF SMALL METALLIC PARTICLES. Journal de Physique Colloques, 1977, 38 (C2), pp.C2-129-C2-132. 10.1051/jphyscol:1977226 . jpa-00217065

\section{HAL Id: jpa-00217065 https://hal.science/jpa-00217065}

Submitted on 1 Jan 1977

HAL is a multi-disciplinary open access archive for the deposit and dissemination of scientific research documents, whether they are published or not. The documents may come from teaching and research institutions in France or abroad, or from public or private research centers.
L'archive ouverte pluridisciplinaire HAL, est destinée au dépôt et à la diffusion de documents scientifiques de niveau recherche, publiés ou non, émanant des établissements d'enseignement et de recherche français ou étrangers, des laboratoires publics ou privés. 


\title{
ON THE STATISTICAL PROPERTIES OF THE ELECTRONIC LEVELS OF SMALL METALLIC PARTICLES

\author{
J. BAROJAS, E. COTA
}

Departamento de Física, Universidad Autónoma Metropolitana, Iztapalapa Apartado Postal 55-534, México 13, D.F.

\section{E. BLAISTEN-BAROJAS, J. FLORES (**) and P. A. MELLO (**)}

Instituto de Física, Universidad Nacional Autónoma de México Apartado Postal 20-364, México 20, D.F.

\begin{abstract}
Résumé. - On examine dans ce travail les arguments de Kubo et Gor'kov-Eliashberg concernant les fluctuations des niveaux d'énergie des particules métalliques. Nous confirmons que la Théorie des Matrices Aléatoires n'est pas adéquate pour décrire le comportement de ces particules quand on leur associe le modèle des électrons libres avec conditions aux limites de Dirichlet. Dans le cadre d'un modèle à deux dimensions exactement soluble on détermine la répartition des écarts entre niveaux d'énergie et on ne constate pas de répulsion entre ces niveaux. Finalement on calcule numériquement la variation de la chaleur spécifique $C_{v}$ en fonction de la température pour une collection de spectres dont la loi de répartition des écarts d'énergies est aléatoire.
\end{abstract}

\begin{abstract}
The arguments of Kubo and Gor'kov-Eliashberg concerning the level fluctuations of small metallic particles (s.m.p.) are reviewed. We make plausible that Random Matrix Theory is not applicable to be s.m.p. problem within the free electron picture and when Dirichlet boundary conditions are used. Next, in the frame of an exactly soluble two-dimensional model, we determine the level-spacing-distribution which shows no level repulsion. Finally we calculate numerically the specific heat $C_{\mathrm{v}}$ at finite temperature for an assembly of spectra following the random distribution law.
\end{abstract}

1. Introduction. - Surface effects are fundamental in determining the thermodynamic properties of small systems, such as small metallic particles (s.m.p.). This is particularly so at low temperatures, where their anomalous thermodynamic behaviour is related to the scarce number of electrons excited above the Fermi level and to the discrete character of the spectrum. In fact, different theories of small metallic particles are related to the different spacing distributions assumed for the one-electron energy levels. The first theoretical attempt to understand the thermodynamic properties of an assembly of s.m.p. is due to Fröhlich [1], who assumed equally spaced levels, thereby obtaining an exponential temperature dependence for the specific heat $C_{v}$ at low temperature $T$. Several years later, Kubo [2] assumed, on the grounds that surface irregularities remove all systematic spectral properties, a completely random sequence of levels, to which corresponds a nearest-neighbour spacing distribution of the Poisson type :

$$
p_{\mathrm{p}}(\chi)=\mathrm{e}^{x}
$$

here $\chi=s / \delta$, where $s$ is the spacing between nearest neighbours and $\delta$ its average value. Using

(*) An extended version of this paper will be published elsewhere.

(**) Consultant of the Instituto Nacional de Energía Nuclear, México. eq. (1), Kubo obtained a linear $T$-dependence of $C_{\mathrm{V}}$ at low temperatures. Later on, Gor'kov and Eliashberg [3] (G.E.) made the assumption that surface irregularities would induce matrix elements between the single-electron wave functions ; since the irregularities are located at random, the matrix elements would also be random numbers, in such a way that the assembly of s.m.p. would be represented by an ensemble of random matrices. The specific character of the ensemble is determined by the symmetries of the Hamiltonian ; in any case, Poisson's law (1) does not hold. On the contrary, the conventional ensembles of random matrices [4] lead to spacing distributions which show level repulsion, that is, $p(\chi) \rightarrow 0$ when $\chi \rightarrow 0$. In particular, the Gaussian orthogonal ensemble gives rise to a distribution which is closely approximated by Wigner's distribution [5] ( $\left.{ }^{1}\right)$,

$$
p_{w}(\chi)=\frac{\pi \chi}{2} \mathrm{e}^{-\pi \chi^{2 / 4}} \text {. }
$$

(1) There are several instances in which the spacing distribution lies between those corresponding to eqs. (1) and (2). In this case, a very appropriate measure [6] of the level repulsion is provided by the parameter $\omega$, obtained through a least-squares fit of the spacing histogram to the function

$$
p_{\omega}(\chi)=a_{\chi} \mathrm{e}^{-b_{\chi} \omega+1}
$$

where $a$ and $b$ are constants so that $p_{\omega}(\chi)$ is normalized and the average value of $\chi$ equals 1 . Notice that eq. (1) corresponds to $\omega=0$ and eq. (2) to $\omega=1$. 
When this is the case for the spacing distribution of levels around the Fermi level, $C_{\mathrm{v}}$ varies as $T^{2}$ for $T \rightarrow 0$.

When G.E. introduced the idea of an ensemble of random matrices as an appropriate model for the s.m.p. problem, they implicitly made two assumptions : first, that the effect of a surface irregularity or boundary perturbation can be dealt with as a matrix problem and, second, that these irregularities indeed give rise to level repulsion in the spectra.

It is the purpose of the present work to analyze the two assumptions mentioned above. In section 2 it is made plausible that one cannot, in general, formulate the problem of boundary perturbations as a matrix one. In section 3 we obtain the spacing distribution for a two-dimensional model which is exactly soluble, showing that a completely random sequence of levels emerges, as first assumed by Kubo [2]. Finally, in section 4, we give the result of a numerical calculation for the specific heat of an assembly of spectra obeying the random level spacing distribution law (1).

2. The eigenvalue problem. - In the free-electron picture, one considers each electron as being enclosed in a closed region $R^{\prime}$ with impenetrable walls $S^{\prime}$. One then has to solve the free Schrödinger equation

$$
\left(\nabla^{2}+k_{m}^{2}\right) \phi_{m}=0
$$

with the boundary condition

$$
\phi_{m}\left(S^{\prime}\right)=0
$$

Assuming these solutions to be known, one would like to solve, in terms of them, the problem for a new boundary $S$ deformed with respect to $S^{\prime}$ and contained therein [7]. The boundary condition to be satisfied on $S$ is again of the Dirichlet type (5). One way to pass from the old problem to the new one is to add to the kinetic energy of eq. (4) an extra potential which is zero everywhere, except in the region between $S$ and $S^{\prime}$, where it takes the value $V_{0}$ : when $V_{0}$ grows, the wave functions become smaller and smaller in this region and they eventually vanish here when $V_{0} \rightarrow \infty$. The matrix elements of this potential between the original wave functions $\phi_{n}$ are then infinite. Therefore, a matrix formulation of the Dirichlet problem with a perturbed boundary is not possible. Notice that this argument does not hold true for Neumann boundary conditions [7].

As an example [8, 9], consider the one'dimensional case in which the unperturbed problem is defined in the interval $[0,1]$ with eigenvalues $\dot{k}_{m}^{2}$ and eigenfunctions $\phi_{m}$, and the perturbed one in the interval $[0,1-\varepsilon], \varepsilon>0$, with eigenvalues $K_{m}^{2}$ and eigenfunctions $\psi_{m}$, which are zero in the interval $[1-\varepsilon, 1]$. The matrix elements of the operator $\mathscr{H}=\sum_{m=1}^{\infty}\left|\psi_{m}>K_{m}^{2}<\psi_{m}\right|$ with respect to $\phi_{r}$ and $\phi_{s}$ are then

$$
\begin{aligned}
& \left\langle\phi_{r}|\mathscr{H}| \phi_{s}\right\rangle= \\
& \quad=\frac{4}{1-\varepsilon} \sin [\pi r(1-\varepsilon)] \sin [\pi s(1-\varepsilon)] \times \\
& \quad \times \sum_{m=1}^{\infty} \frac{m^{4}}{\left[m^{2}-(1-\varepsilon)^{2} r^{2}\right]\left[m^{2}-(1-\varepsilon)^{2} s^{2}\right]}
\end{aligned}
$$

In fact, the series is not convergent.

We therefore see that the arguments of G.E. do not justify the application of R.M.T. to represent an assembly of particles with impenetrable walls.

3. Spectrum fluctuations in a simple model. Because of the difficulties to relate the spacing distribution of the s.m.p. problem with a problem of known solution, like conventional R.M.T., we shall now consider a very simple model, whose eigenvalues are known and can thus be analyzed to find directly the spacing distribution.

The model consists of $N$ two-dimensional rectangles, the $\alpha$-th one containing $n^{(\alpha)}$ free electrons ; the rectangle $\alpha$ has dimensions 1 and $1-\varepsilon^{(\alpha)}$. To define the assembly of rectangles, or platelets, $\varepsilon^{(\alpha)}$ is varied; in particular $\varepsilon^{(\alpha)}$ could be a random variable, although this is not at all necessary for what follows. With these definitions, and under Dirichlet boundary conditions, the single-electron spectrum for the $\alpha$-th platelet is

$$
E_{n_{1} n_{2}}^{(\alpha)}=\frac{\hbar^{2} \pi^{2}}{2 m_{\mathrm{e}}}\left[\frac{n_{1}^{2}}{\left(1-\varepsilon^{(\alpha)}\right)^{2}}+n_{2}^{2}\right] .
$$

We can now study the spectrum fluctuations of this model in two different ways : first, fixing $\varepsilon^{(\alpha)}$, we analyze the nearest-neighbour spacing distribution along a single spectrum; and, second, we calculate the distribution, along the assembly of rectangles, of the energy difference between the Fermi level and the one immediately above it. In both cases the resulting distribution is approximately the same : the parameter $\omega$ of eq. (3) has the value $0.023 \pm 0.035$ for $\varepsilon^{(\alpha)}=0.015$ and the value $0.003 \pm 0.05$ throughout the ensemble. Essentially the same result has been obtained for platelets with $n^{(\alpha)} \sim 10^{3}$ and with $n^{(\alpha)} \sim 10^{4}$ electrons.

Since $p(\chi)$ is the same along one particular spectrum as well as across the ensemble, we say that the model is ergodic with respect to the nearest-neighbour spacing distribution. But, what is more important, $p(\chi)$ follows rather closely the Poisson law and does not agree with any of the 
predictions of the various ensembles normally used [10] in R.M.T., all of which show the level repulsion phenomenon. The original assumption of Kubo [2] seems to be more appropriate, at least as far as the present model is concerned.

4. Calculation of the specific heat. - Assuming a completely random sequence of levels and constant level density, Kubo [2] obtained the specific heat $C_{\mathrm{v}}$ at low temperatures up to second order in $k_{\mathrm{B}} T / \delta$, where $k_{\mathrm{B}}$ is Boltzmann's constant. We shall now extend his result to higher values of $k_{\mathrm{B}} T / \delta$ by computing numerically the partition function $Z$ for an assembly of $N$ single-electron spectra constructed with a random number generator in such a way that the Poisson law (1) is followed exactly with $\delta=1$ throughout the whole spectrum. Notice that this assembly of spectra can be considered as a generalization of the assembly dealt with in section 3 . is

The partition function for the assembly of spectra

$$
Z=\prod_{\alpha=1}^{N} \sum_{\lambda} g_{\lambda} \exp \left(-\mathscr{E}_{\lambda}^{(\alpha)} / k_{\mathrm{B}} T\right)
$$

where $g_{\lambda}$ and $\mathscr{E}^{(\alpha)}$ are, respectively, the level degeneracies and energies of the $\alpha$-th particle. In order to compute (8) numerically we take into account that at low temperatures only a small number of states of the many-body system give a significant contribution to the specific heat. These states are packed into a band around the Fermi level, its width being of the order of $k_{\mathrm{B}} T$. As any actual calculation is always done within a certain accuracy, the width of the excitation band at a given temperature is fixed by the numerical accuracy. Under these conditions, the accessible configurations of the assembly are generated by the computer applying a combinatorial technique. The specific heat is given by

where

$$
\frac{C_{\mathrm{v}}}{k_{\mathrm{B}}}=\frac{1}{N}\left(\frac{1}{k_{\mathrm{B}} T}\right)^{2} \sum_{\alpha=1}^{N} \frac{S_{0}^{(\alpha)} S_{2}^{(\alpha)}-\left[S_{1}^{(\alpha)}\right]^{2}}{\left[S_{0}^{(\alpha)}\right]^{2}}
$$

$$
S_{i}^{(\alpha)}=\sum_{\lambda}\left[\mathscr{E}_{\lambda}^{(\alpha)}\right]^{l} \exp \left(-\mathscr{E}_{\lambda}^{(\alpha)} / k_{\mathrm{B}} T\right) .
$$

The numerical results for even and odd number of electrons are condensed in figure 1. The dots correspond to the average values and the error bars indicate the standard deviation for a sample of 1000 spectra. These results are compared with Kubo's expansion of $C_{\mathrm{v}}$ up to second order in $k_{\mathrm{B}} T / \delta$, shown in the figure with dashed lines. The numerical results for $C_{\mathrm{v}}$ deviate from Kubo's approximation when $k_{\mathrm{B}} T / \delta \geqslant 0.025$. This is so because at higher temperatures more many-electron configurations, not taken into account in Kubo's calculation, start to be important. Also shown in the

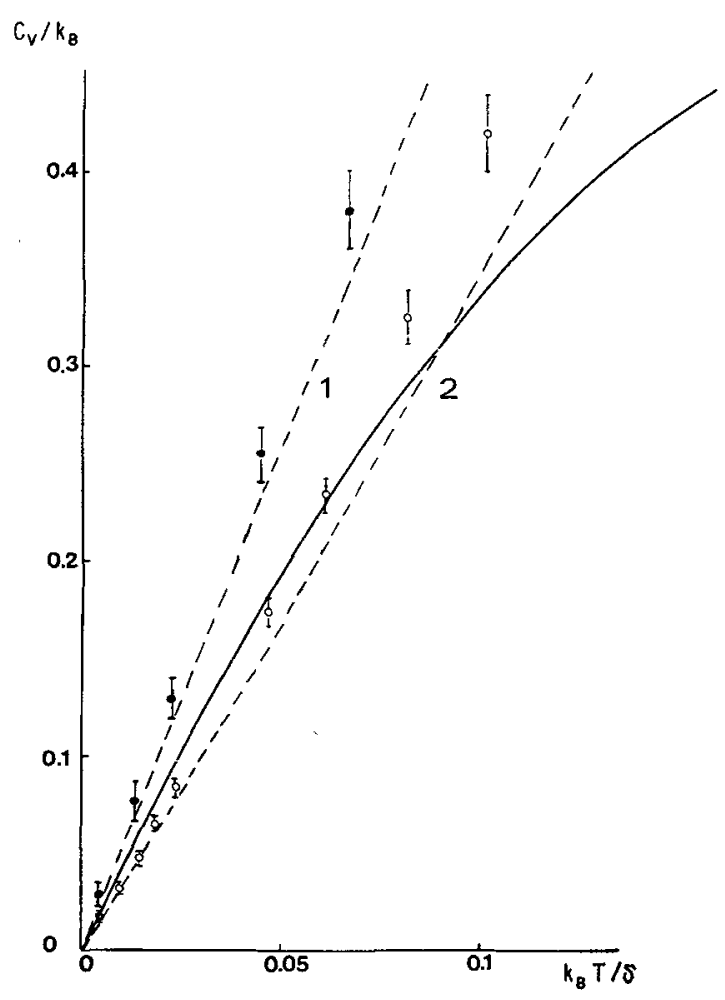

Fig. 1. - Numerical computation of the specific heat $C_{v} v s$. temperature. Full and empty dots stand for samples of 1000 spectra with even and odd number of electrons, respectively. Curves 1 and 2 indicate Kubo's [2] approximation for even and odd number of electrons, respectively. The solid line corresponds to Denton et al. [11] approximation for the even-odd average.

figure with full line is the even-odd average calculated by Denton et al. [11].

5. Conclusions. - Several points emerge from this paper. First, R.M.T. is not applicable to the problem of boundary perturbations with Dirichlet conditions, since this problem cannot be formulated in matrix form. Second, the analysis of a simple two-dimensional model tells us that the spacing distributions of an assembly of small particles are not of the type encountered in R.M.T. but rather of a Poisson nature. This is contrary to what is normally believed, even on the grounds of experimental evidence concerning the magnetic susceptibility of small metallic particles. This evidence is, however, scanty and somewhat unreliable at very low temperatures, since impurities and other effects could play a significant role [12].

In any case, assuming a Poisson spacing distribution for an assembly of $N$ spectra, we computed numerically an exact value for the specific heat. The calculation shows that Kubo's original analytical result is correct only for $k_{\mathrm{B}} T / \delta<0.025$, a rather small value. 


\section{References}

[1] FröHLICH, H., Physica 4 (1937) 406.

[2] Киво, R., J. Phys. Soc. Japan 17 (1962) 975.

[3] Gor'kov, L. P., Eliashberg, G. M., Sov. Phys. JETP 21 (1965) 940.

[4] Metha, M. L., Random Matrices and Statistical Theory of Energy Levels (Academic Press, New York) 1967, and references therein.

[5] Wigner, E. P., Gatlinburg Conference on Neutron Physics, ORNL 2309 (1957) 59.

[6] Brodx, T. A., Nuovo Cimento Lett. 7 (1973) 482.

[7] Morse, P., Feshbach, H., Methods of Theoretical Physics (McGraw-Hill, New York) 1953, Chapter IX.
[8] Cota, E., thesis, Universidad Nacional Autónoma de México, (1975).

[9] Barojas, J., Cota, E., Blatsten-Barojas, E., Flores, J., Mello, P. A., to be published.

[10] Kubo, R., Polarisation, Matière et Rayonnement, Livre Jubilé en l'honneur du Pr. A. Kastler (Presses Universitaires, Paris) 1969.

[11] Denton, R., Muhlschlegel, B., Scalapino, D. J., Phys. Rev. B 7 (1973) 3589.

[12] Yee, P., Knight, W. D., Phys. Rev. B 11 (1975) 3261, and references 3 and 4 therein. 